\title{
Expression and Sequence Analysis of CYP86B1 in Blackberry (Rubus Spp.)
}

\author{
Xunju Liu ${ }^{1}$, Dan Jiang ${ }^{1}$, Yan Wang ${ }^{2}$, Haoru Tang ${ }^{2}$ and Qing Chen ${ }^{1, a, *}$ \\ ${ }^{1}$ College of Horticulture, Sichuan Agricultural University, Sichuan, China; \\ ${ }^{2}$ Institute of Pomology and Olericulture, Sichuan Agricultural University, S \\ a supnovel@gmail.com
}

Keywords: Rubus spp, CYP86B1, Cloning and expression.

\begin{abstract}
To understand the molecular mechanisms of cuticle wax formation in blackberry, the waxy synthetic related gene CYP86B1 was cloned from blackberry. The open reading frame of this gene was $1692 \mathrm{bp}$, encoding 563 amino acid residues with a molecular weight of $64.5 \mathrm{kD}$ and a theoretical isoelectric point of 7.31. Its amino acid sequence has the highest identity with strawberry. Real-time PCR results showed that the expression of CYP86B1 gene was significantly decreased with the accompanied by fruit ripening.
\end{abstract}

\section{Introduction}

Plant epidermal wax is located on the outermost layer of plant cells, it plays an important role in responding to plant adaptations to biotic stresses or abiotic stresses in regulating plant growth and development. It prevents plants from being exposed to excessive UV radiation by limiting the plant's non-stomatal water loss; it affects the plant-insect and plant-pathogen interactions; it also works with plant self-cleaning, waterproof function is closely related [1]. In plant growth and development, plant epidermal wax directly affects plant epidermal morphogenesis, especially the development of specialized cells; plant epidermal wax can ensure the normal demarcation of plant organs and prevent plant organ fusion; plant waxy layer can affect pollen grains surface structure and the structure of the oil-bearing layer, controlling the hydration of pollen, thereby affecting plant fertility [2].

Cytochrome P450 (CYP450) represents a large family of heme-oxidizing heme proteins that belong to the category of monooxygenases and are named for their specific absorption at $450 \mathrm{~nm}$. It exists in all organisms and is involved in the metabolism of both internal and external substances. A large number of previous studies have found CYP86A subfamily genes encoding fatty acid $\omega$-hydroxylase [3], members of the CYP86B subfamily have $45 \%$ homology with members of the CYP86A subfamily, may suggest that CYP86B1 and CYP86A1 metabolic pathways involved in similar. In 2009, Compagnon V found CYP86B1 is a long-chain fatty acid hydroxylase and it is localized in the endoplasmic reticulum and participates in the biosynthesis of polyester monomers during plant growth [4]. The CYP86B1 gene is mainly expressed in plant roots and seeds [5]. Subcellular localization results show that CYP86B1 is mainly deposited on the tapetum cells and guard cells of Arabidopsis anthers [6]. In addition, few studies on the CYP86B1 gene, CYP86B1 gene in the population is relatively conservative? What are the factors related to the expression of CYP86B1 gene? This experiment intends to understand the bioinformatics characteristics of CYP86B1 gene and its expression in different developmental stages for provide the basis for further study of CYP86B1 gene.

Blackberry is a widely cultivated, highly edible and medicinal value specialty economic fruit tree. During the growth of the blackberry, the climate during the fruit development period was hot. The surface of the fruit was covered with a large amount of white waxy layer to resist intense sunburn. Because of this trait, the blackberry has become a suitable material for studying plant wax formation. In this study, the spineless blackberry 'Arapaho' was used as a material. From the transcriptomic data from previous research in our group, the transcript sequence homologous to Arabidopsis CYP86B1 was extracted. Gene-specific primers were designed and the CYP86B1 gene was cloned by RT-PCR. 
The sequence of CYP86B1 gene in blackberry was analyzed by bioinformatics method. The expression of CYP86B1 gene in blackberry was analyzed by qRT-PCR. Prediction of CYP86B1 gene in the formation of blackberry waxy important role in revealing plant epidermal waxy and environmental interaction mechanism and breeding varieties provide theoretical basis for resistance.

\section{Materials and Methods}

\subsection{Materials}

In April of 2015, the young leaves and fruits of blackberry cultivar 'Arapaho', which are planted at the teaching and scientific research base of Sichuan Agricultural University were taken as materials and frozen in liquid nitrogen for RNA extraction. Fruits of three maturity level were included: young fruits (5 days post anthesis, DPA), red fruits (20 DPA), and black fruits (25 DPA). Fruits of the same developmental stage were randomly selected on different branches of the same shrub. 20 Fruits were randomly selected as one biological replicate. Three replicates were conducted for each stage.

\subsection{CYP86B1 Gene Cloning and Sequencing}

The young leaves, three maturity fruits were powdered in liquid nitrogen. Total RNA was extracted by modified CTAB method [7]. cDNA was synthesized according to the instructions of the Reverse Script kit Prime Script RT reagent Kit with gDNA Eraser. From the transcriptome data of our previous research (Bioproject PRJNA401210), a transcript homologous to Arabidopsis thaliana CYP86B1 was extracted. Two specific primers were designed in Primer 5.0 software (CYP86B1-F: AGACTACATACAACTCCATCAT and CYP86B1-R; ATCCATGATGAACACCAATCTCACC, the underline is the start codon).

PCR amplification was conducted in $20 \mu \mathrm{L}$ reaction mixture, PCR products were detected by $1 \%$ agarose gel electrophoresis. After the recovering from the gel, the specific PCR amplicon was ligated into pEASY-Blunt vector and transformed into E. coli Trans1-T1. After overnight culture and single colonies were picked for detection. The positive clones were sent to Sangon Biotech for sequencing.

\subsection{Bioinformatic Analysis}

The open reading frame was searched with NCBI ORF finder. Protein molecular weight and theoretical isoelectric point were analyzed with ProtParam. Conserved domains were screened by NCBI-CDD. Multiple sequence alignments were performed with MAFT. Phylogenetic tree was constructed by IQTREE.

\subsection{Real-time Quantitative Analysis of CYP86B1 Gene Expression}

The blackberry actin gene was used as an internal reference (Qactin-F: AGACTACATACAACT CCATCAT and Qactin-R: ACCAATCCAGACACTATACTT). The RT-PCR primer was designed based on the sequenced HTH gene sequence, Qcyp-F: TCGCCTCTGCTTGGGAAA and Qcyp-R: CTCTGGTGATGATGACGCCTAT. The expression of CYP86B1 gene in different developmental stages of fruit was detected by total RNA of blackberry.

RT-qPCR reaction mixture consists of SYBR Green fluorescent dye $10 \mu \mathrm{L}$, the upstream and downstream primers $0.8 \mu \mathrm{L}$, cDNA $1.5 \mu \mathrm{L}$, dd $\mathrm{dd}_{2} \mathrm{O}$ to $20 \mu \mathrm{L}$. Reaction were conducted at $95{ }^{\circ} \mathrm{C}$ for 3 min, followed by 40 PCR cycles of $95^{\circ} \mathrm{C} 10 \mathrm{~s}, 55^{\circ} \mathrm{C} 30 \mathrm{~s}, 72{ }^{\circ} \mathrm{C} 15 \mathrm{~s}$. Fluorescence was collected at the third step of each cycle. Reactions with no template cDNA were used as negative controls. All PCR reactions were replicated three times, and the data were analyzed by $2^{-\Delta \Delta C t}$ method. The relative expression values were subjected to One-way ANOVA analysis in SPSS software (v20.0).

\section{Results}

\subsection{Cloning of CYP86B1 Gene}

Selected blackberry leaves, using modified $\mathrm{CTAB}$ method to extract total RNA, reverse transcribed into cDNA. PCR products were recovered, TA cloning, bacterial PCR identification, plasmid extraction, sent to the company sequencing to obtain full-length CYP86B1 sequence. 


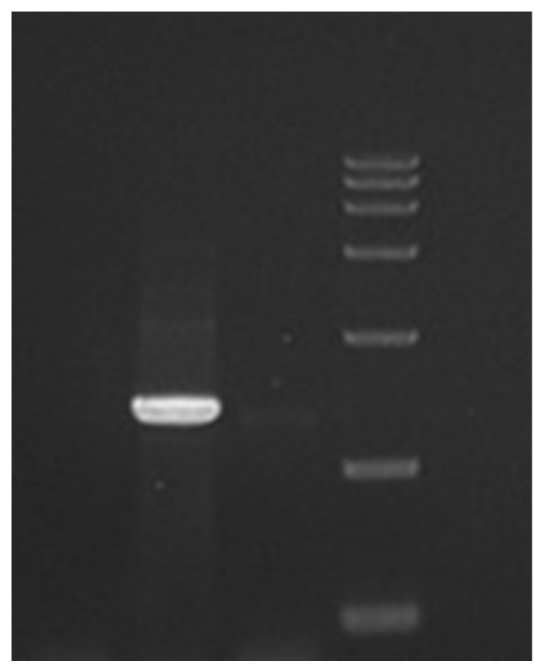

$2500 \mathrm{bp}$

$1000 \mathrm{bp}$

Fig. 1 1\% agarose gel electrophoresis of CYP86B1 identification of bacterial fluid by PCR

\subsection{CYP86B1 Gene in Rubus Spp.}

The CYP86B1 gene homologous to Arabidopsis CYP86B1 was successfully identified in Rubus spp. The open reading frame of this gene was $1692 \mathrm{bp}$, encoding a peptide of 563 amino acid residues with a molecular weight of $64.5 \mathrm{kD}$. Theoretical isoelectric point is 7.31. Multiple sequence alignment was conducted with the currently identified other CYP86B1 proteins (Fig. 2).

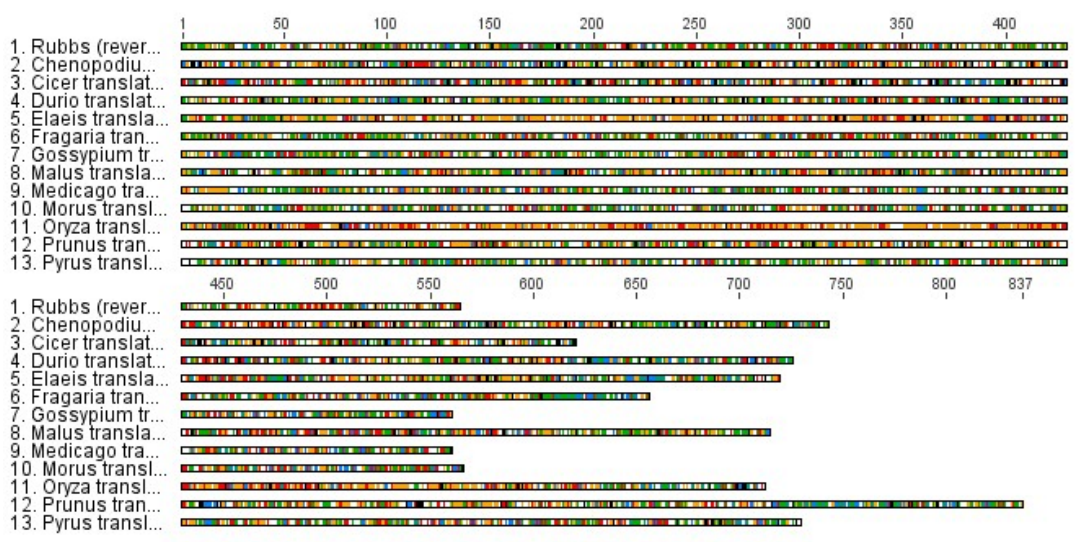

Fig. 2 Sequence alignment of CYP86B1 related proteins.

\subsection{Phylogeny of CYP86B1 Protein}

The phylogeny of CYP86B1 related proteins from species like Malus, Pyrus, and Citrus were referred by most likelihood (ML) trees (Fig. 3). The blackberry CYP86B1 protein has the highest similarity with the amino acid sequence of strawberry CYP86B1 protein, indicating the closest evolutionary relationship with strawberry.

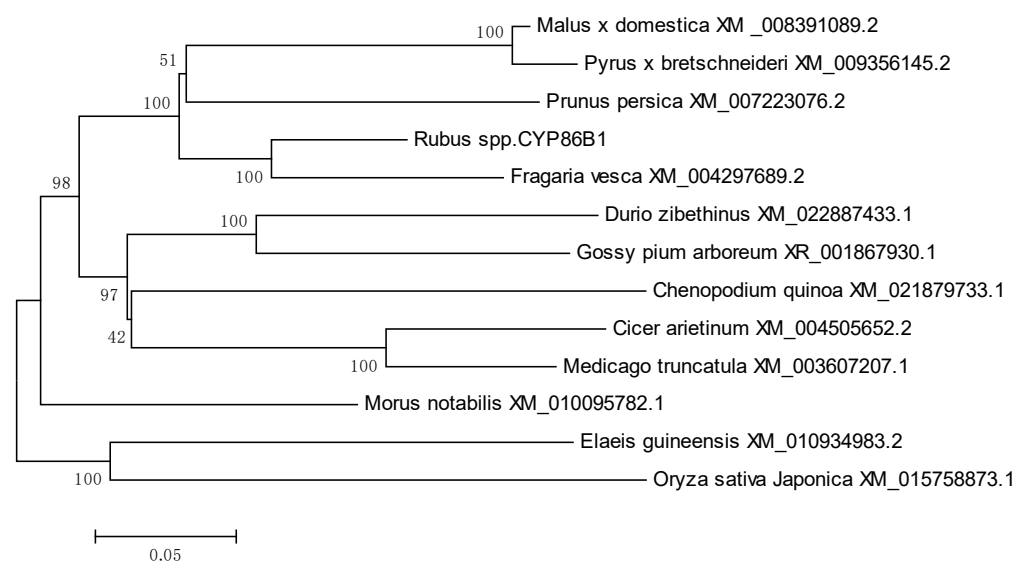

Fig. 3 ML tree of CYP86B1 of blackberry and other plants. 


\subsection{CYP86B1 Gene Expression in Different Developmental Stages of Fruit}

The RT-qPCR was used to access the expression of CYP86B1 gene during blackberry fruit development. As is depicted in Fig. 4, the expression level of CYP86B1 gene decreased with fruit ripening. This indicates it may be involved in the early development of blackberry fruit.

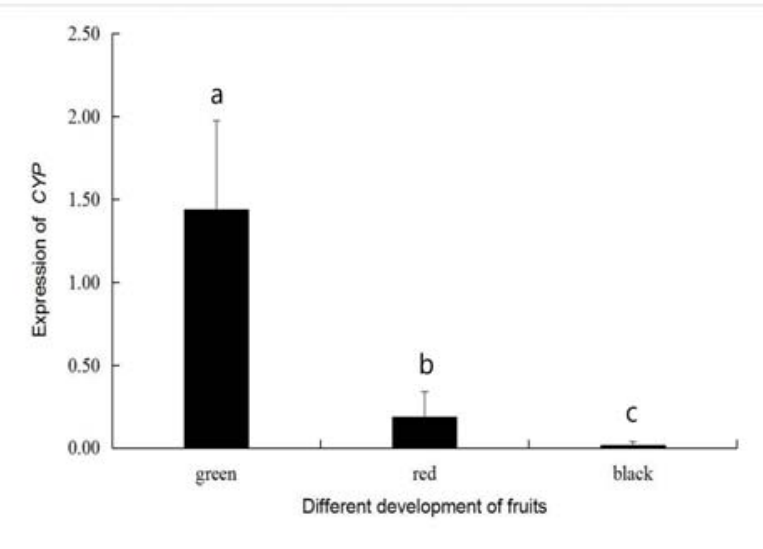

Fig. 4 Expression profiling of CYP86B1 gene in different fruit development stages. Same letters indicate non-significant differences among stages (One-way ANOVA, $p<0.05$ ).

\section{Conclusion}

Plant epidermal wax components are more complex, but in many species, different growth stages, epidermal wax components of different organs, very long chain aliphatic compounds and their derivatives are ubiquitous [8,9]. In this experiment, the obtained CYP86B1 protein sequence of the blackberry has high similarity with the CYP86B1 protein sequence of other species, indicating that the CYP86B1 gene may be relatively conservative in the population. The results of real-time PCR showed that the CYP86B1 gene was expressed at different stages of fruit development, and most likely involved in the synthesis and expression of CYP86B1 genes during the development of black berry As the fruit mature, CYP86B1 gene expression decreased significantly. But what factors will affect the expression of CYP86B1 gene needs further study.

\section{References}

[1]. Fich E A, Segerson N A, Rose J K. The Plant Polyester Cutin: Biosynthesis, Structure, and Biological Roles [J]. Annual Review of Plant Biology, 2016, 67(1):207.

[2]. Kosma D K, Bourdenx B, Bernard A, et al. The impact of water deficiency on leaf cuticle lipids of Arabidopsis. [J]. Plant Physiology, 2009, 151(4):1918.

[3]. Voisin D, Nawrath C, Kurdyukov S, et al. 2009. Dissection of the complex phenotype in cuticular mutants of Arabidopsis reveals a role of SERRATE as a mediator. PLOS Genet.5:e1000703

[4]. Compagnon V, Diehl P, Benveniste I, et al. CYP86B1 is required for very long chain $\omega$-hydroxyacid and $\alpha, \omega$-dicarboxylic acid synthesis in root and seed suberin polyester [J]. Plant physiology, 2009, 150(4): 1831-1843.

[5]. Ho“ fer R, Briesen I, Beck M, et al. 2008. The Arabidopsis cytochrome P450 CYP86A1 encodes a fatty acid v-hydroxylase involved in suberin monomer biosynthesis. J Exp Bot 59: 2347-2360

[6]. Franke R, Ho“ fer R, Briesen I, et al. 2009. The DAISY gene from Arabidopsis encodes a fatty acid elongase condensing enzyme involved in the biosynthesis of aliphatic suberin in roots and the chalaza-micropyle region of seeds. Plant J 57:80-95 
[7]. Chen Q, Yu H, Wang X R, et al, An alternative cetyltrimethyl ammonium bromide-based protocol for RNA isolation from blackberry (Rubus L.)[J]. Genetics and Molecular Research, 2012, 11(2):1773-1782.

[8]. Javelle M, Vernoud V, Rogowsky PM, et al. 2011. Epidermis: the formation and functions of a fundamental plant tissue. New Phytologist, 189(1): 17-39.

[9]. Jetter R, Kunst L. 2008. Plant surface lipid biosynthetic pathways and their utility for metabolic engineering of waxes and hydrocarbon biofuels. Plant Journal, 54(4): 670-83. 